\title{
Signatures of the continuum electron phase in molecular strong-field photoelectron holography
}

\author{
M. Meckel ${ }^{1,2}$, A. Staudte ${ }^{2}$, S. Patchkovskii ${ }^{3}$, D. M. Villeneuve ${ }^{2}$, P. B. Corkum², R. Dörner ${ }^{1}$ \\ and M. Spanner ${ }^{3 \star}$
}

\begin{abstract}
Laser-driven electron recollision is at the heart of the rapidly growing field of attosecond science. The recollision wavepacket is qualitatively described within the strong-field approximation, which commonly assumes tunnelling ionization and plane-wave propagation of the liberated electron in the continuum. However, with increasing experimental sophistication, refinements to this simple model have become necessary. Through careful modelling and measurements of laser-induced recollision holography using aligned $\mathbf{N}_{\mathbf{2}}$ molecules, we demonstrate that the continuum electron wavepacket already carries a non-trivial spatial phase structure immediately following ionization. This effect is of rather general character: any molecule and any non-isotropic system that is ionized by a strong laser field will exhibit an offset in the phase of the continuum electron wavepacket. Specifically, this has important implications for any coherent scattering process in molecules, such as highharmonic generation or laser-induced electron holography.
\end{abstract}

E lectron holography is a well-established technique in electron microscopy ${ }^{1}$. An electron wave produced at a cathode and accelerated towards a microscopic object can produce a hologram of this object through the interference of its scattered and unscattered components ${ }^{2}$. Recently, it has been shown that laserdriven electron recollision ${ }^{3}$ can produce holographic interference patterns in the photoelectron momentum distribution of ionized atoms ${ }^{4}$. In direct analogy to the original idea ${ }^{2}$, the atom serves as its own photocathode and the electron wave is being accelerated by the oscillating electric field of the laser back onto its parent ion, where it can elastically scatter. Owing to the oscillating nature of the accelerating field, multiple scattering and hence various levels of holographic interference will occur ${ }^{5-8}$. Recollision energies of typically below $100 \mathrm{eV}$ have so far prevented recollision-based holography from providing information about the parent ion'. Instead, holography has been shown to yield insight into the recollision process itself ${ }^{7}$.

The strong-field approximation of recollision has been extremely successful in modelling non-sequential double ionization ${ }^{10}$, highharmonic generation (HHG; ref. 11) and sub-femtosecond pulse generation-the hallmarks of attosecond science ${ }^{12}$. However, a number of corrections to the original strong-field approximation (SFA; ref. 13) have become necessary as the experimental precision increased. First, the effect of the ionic binding potential during the continuum propagation of the electron wavepacket was recognized ${ }^{14,15}$. Second, it was found that the nodal structure and symmetries of the particular orbital from which the electron originates strongly affect the amplitudes of the continuum wavepacket $^{16-19}$. Third, it was realized that the continuum wavepacket will be distorted from the plane-wave approximations during the recollision step ${ }^{20-22}$.

Here, we have applied recollision-based electron holography to aligned molecules to study the spatial phase of the recollision wavepacket. We accomplish this by studying the shift of the holographic fringe pattern in the observable electron momentum distributions for different alignment angles. We present a theoretical analysis of the experiment using a reduced-dimensionality softcore two-centre model to identify the origin of the alignment dependence. We find that the dependence does not originate in the scattering event as the electron recollides with the core, but rather in the emission step of the photoelectron. As illustrated in Fig. 1a, when the molecule is at an angle to the electric field polarization, the continuum wavepacket is born with a phase structure that is spatially offset from the molecular origin. This leads to the molecular alignment dependence of the holographic fringes
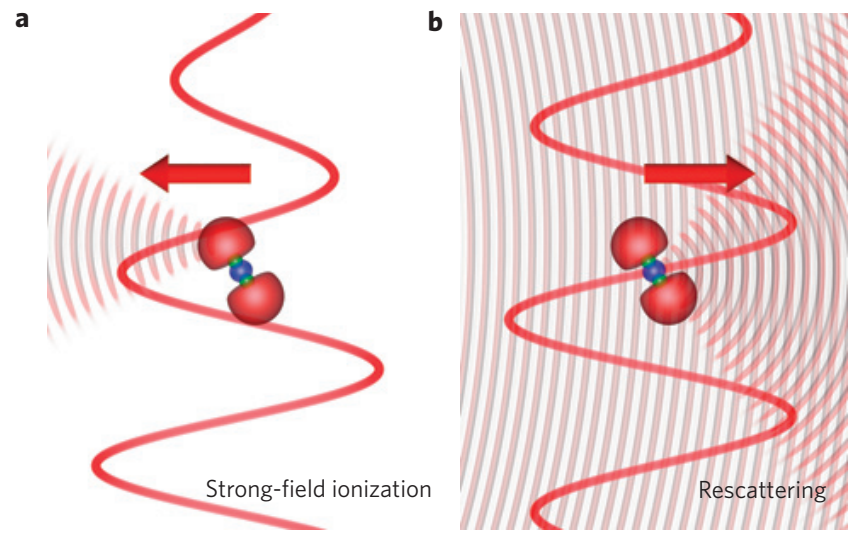

Figure 1 | Artist's impression of the ionizing and recolliding wavepackets. a, In strong-field ionization of an aligned $\mathrm{N}_{2}$ molecule, the electron will be emitted with an offset from the molecular centre. This offset can be larger than the molecular bond length. $\mathbf{b}$, When the electron is driven back to the parent molecule in a strong laser field the spatial offset can modify the recollision process (here, the scattering in the ionic potential)

\footnotetext{
${ }^{1}$ Institut für Kernphysik, Goethe Universität, Max-von-Laue Str. 1, D-60438 Frankfurt, Germany, ${ }^{2}$ Joint Laboratory for Attosecond Science, National Research Council and University of Ottawa, 100 Sussex Drive, Ottawa, Ontario K1A OR6, Canada, ${ }^{3}$ National Research Council, 100 Sussex Drive, Ottawa, Ontario K1A 0R6, Canada. *e-mail: michael.spanner@nrc.ca
} 

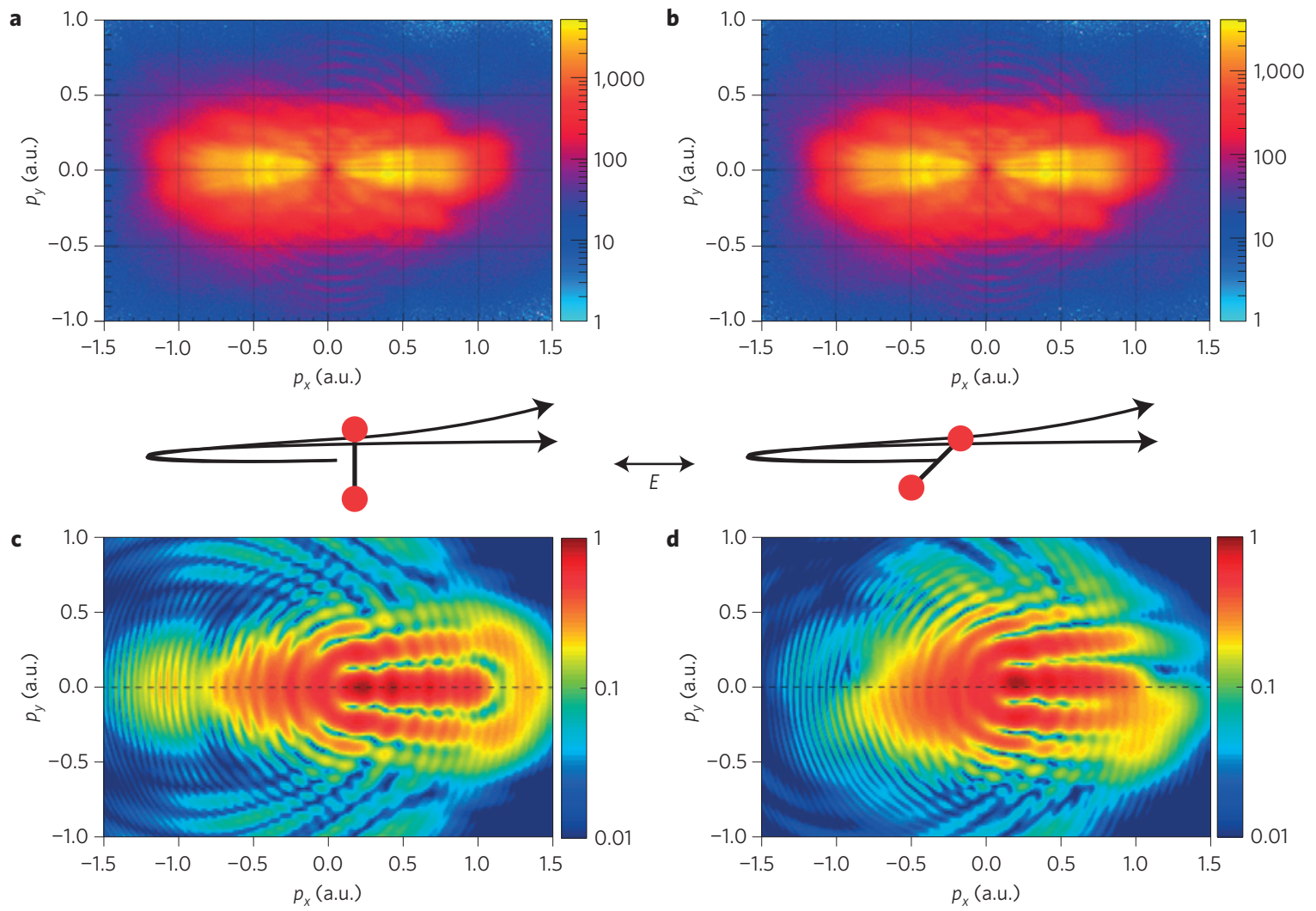

Figure 2 | Measured and simulated photoelectron spectra showing the holographic interference. a, Measured photoelectron momentum distribution (colour scale, a.u.) of $\mathrm{N}_{2}$ molecules aligned along the $y$ axis $\left(\left\langle\cos ^{2} \theta\right\rangle=0.6\right.$ ), perpendicular to the probe pulse polarization along the $x$ axis for $800 \mathrm{~nm}, 40 \mathrm{fs}$ laser pulses with a peak intensity of $I_{0}=1.3 \times 10^{14} \mathrm{~W} \mathrm{~cm}^{-2}$. The integration over $p_{z}$ is limited to an out-of-plane angle $\theta<10^{\circ}$. $\mathbf{b}$, Same as $\mathbf{a}$ for $45^{\circ}$ alignment. c, Simulated photoelectron momentum distribution of a 1.25 cycle, $800 \mathrm{~nm}$ laser field at $I_{0}=1.5 \times 10^{14} \mathrm{~W} \mathrm{~cm}^{-2}$ with horizontal polarization and a model two-centre potential aligned at $90^{\circ}$ to the polarization (see text for details). $\mathbf{d}$, Same as $\mathbf{c}$ for $45^{\circ}$ alignment. Here $\tau=2 \pi / \omega$ is the laser oscillation period.

(Fig. 1b). We confirm that the uncovered mechanism is also present in a $3 \mathrm{D}$ simulation of strong-field ionization of $\mathrm{N}_{2}$. Thus, in our experiment, electron holography provides information about the continuum electron wavepacket rather than the scattering object.

The experiment was performed using COLTRIMS ${ }^{23}$ in a similar experimental set-up to that used in a previous experiment studying laser-induced electron diffraction in $\mathrm{N}_{2}$ (ref. 17). We used a Ti:Sabased, femtosecond laser system, producing $800 \mathrm{~nm}, 40 \mathrm{fs}, 5 \mu \mathrm{J}$ pulses with a repetition rate of $30 \mathrm{kHz}$. The pulses were split in a Michelson interferometer into a $60 \mathrm{fs}$ pump pulse for the nonadiabatic alignment of the molecules and a $40 \mathrm{fs}$ probe pulse with variable delay. The probe pulse was focussed to a peak intensity of $1.3 \times 10^{14} \mathrm{~W} \mathrm{~cm}^{-2}$. Both pulses were linearly polarized. The pump arm of the interferometer had a motorized half-wave plate to align the molecules at any angle with respect to the probing pulse polarization. The laser pulses were focused into a continuous $\mathrm{N}_{2}$, supersonically cooled gas jet. The resulting photoelectrons were detected in coincidence with the $\mathrm{N}_{2}^{+}$ion. The degree of alignment relative to the polarization of the alignment pulse is estimated to be $\left\langle\cos ^{2} \theta\right\rangle=0.6$.

Figure $2 \mathrm{a}, \mathrm{b}$ shows the experimental photoelectron momentum distributions from $\mathrm{N}_{2}$ molecules in the $x-y$ polarization plane, that is, perpendicular to the light propagation direction. Electrons are integrated within a conical cut of $\pm 10^{\circ}$ out of the $x-y$ plane. Figure 2a shows the perpendicular geometry, while Fig. $2 \mathrm{c}$ shows the $45^{\circ}$ geometry. As we will see below, imperfect alignment is the reason that an alignment dependence of the holographic interference cannot be observed in this representation of the data.
To model the experiment we solve the two-dimensional (2D) time-dependent Schrödinger equation (TDSE) for a two-centre soft-core potential (see Supplementary Section II for the specific form of the potential). In the following examples, we use a bond length of $R_{0}=4$ a.u. and a soft-core parameter $a=0.1$ to give an ionization potential of $14.8 \mathrm{eV}$. This bond length is twice as large as the bond length of $\mathrm{N}_{2}$. As demonstrated and explored in Supplementary Section VI, the 2D model at the equilibrium bond length of $\mathrm{N}_{2}$ does not yield holographic fringes with a strong angle dependence. However, we do check that features of the $R_{0}=4$ a.u. model that lead to shifted holographic fringes being present in a full calculation of strong-field ionization of realistic $3 \mathrm{D} \mathrm{N}_{2}$ discussed below. For the present $2 \mathrm{D}$ simulations, the laser intensity was set to $1.5 \times 10^{14} \mathrm{~W} \mathrm{~cm}^{-2}$. In the simulation, we allow for a half-cycle ionization burst, after which we remove the ground state. We then propagate the TDSE for another 0.75 optical cycles. At this point we extract the momentum distribution of the wavefunction. The results are shown in Fig. 2c,d for the alignment angles corresponding to the experiment. In contrast to the experiment we assumed a perfect alignment. The holographic interference appears as horizontal finger-like structures near $p_{y}=0$. In the $45^{\circ}$ case, the holographic interference is shifted and no longer symmetric about $p_{y}=0$.

As the alignment dependence of the experimental photoelectron momentum distribution is very weak, we use a normalization approach, established in ref. 17, to extract the angular variation present in the data. However, whereas in ref. 17 photoelectrons at a pump-probe delay corresponding to antialignment were used for the reference distribution, here we use 

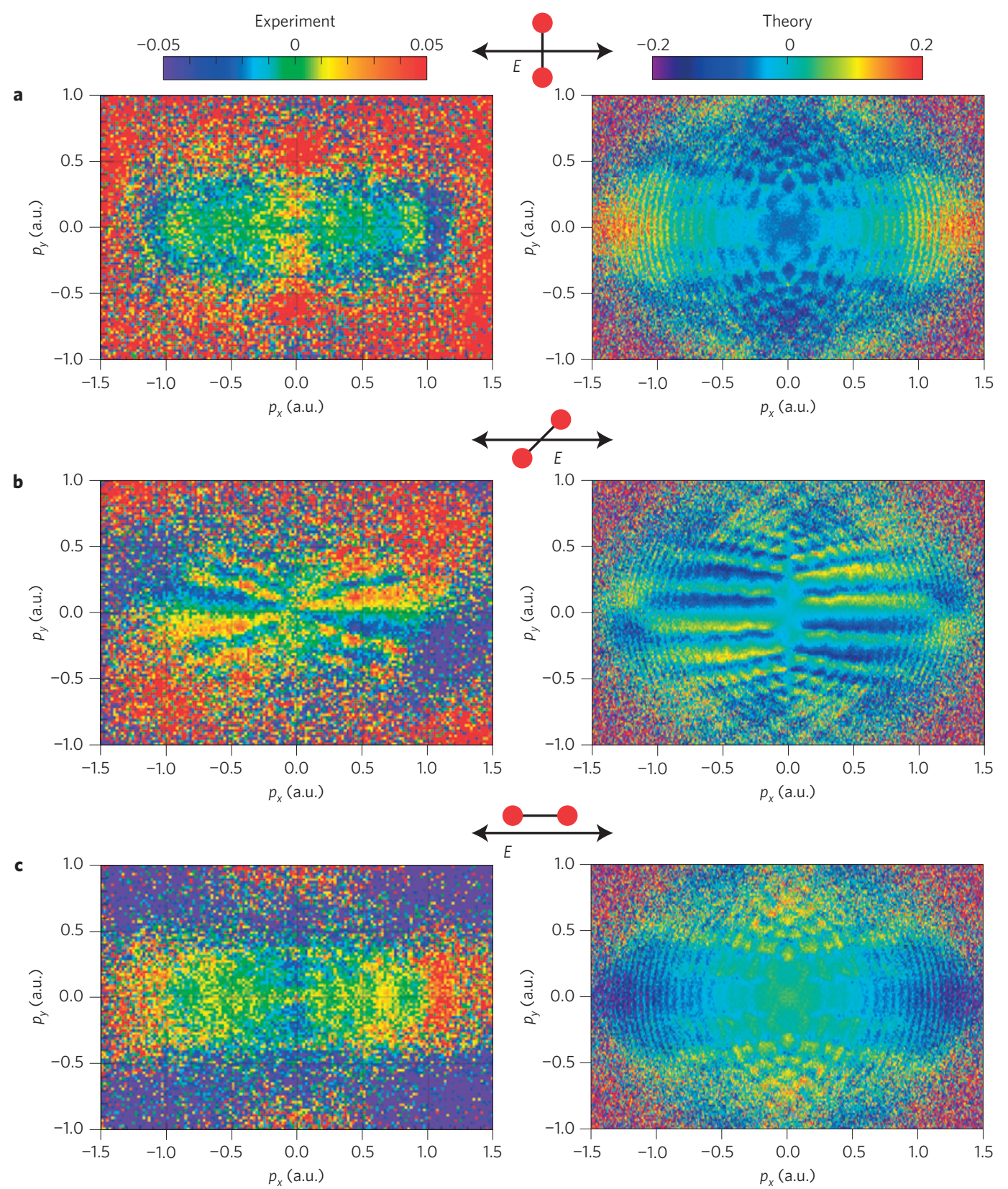

Figure $\mathbf{3}$ | Normalized difference (ND) plots. Left column: experiment, right column: theory, with added uniform random noise (1.5\% amplitude of the maximum of the spectrum). $\mathbf{a}, \mathbf{b}$ and $\mathbf{c}$, are for perpendicular, $45^{\circ}$ and parallel geometries respectively, as depicted by the small cartoon in each panel.

as reference the sum over all distributions for alignments from $0^{\circ}$ to $180^{\circ}$ in $2^{\circ}$ steps. With this reference distribution labelled as $D_{\text {ref }}(\mathbf{p})$, the normalized difference (ND) plots are defined as $N D(\mathbf{p} ; \theta)=\left(D(\mathbf{p} ; \theta)-D_{\text {ref }}(\mathbf{p})\right) /\left(D(\mathbf{p} ; \theta)+D_{\text {ref }}(\mathbf{p})\right)$, where $D(\mathbf{p} ; \theta)$ is the photoelectron distribution corresponding to the alignment angle of $\theta$. Both $D(\mathbf{p} ; \theta)$ and $D_{\text {ref }}(\mathbf{p})$ are normalized such that they sum to 1 when integrated over all momentum space. Figure 3 shows the experimental and simulated ND plots for three alignments, as indicated in the figure. The complete set of the experimental data covering all alignment angles can be found in Supplementary Section I. The simulated ND used the alignment-averaged spectra, as well as a mirroring of the single recollision photoelectron spectra to mimic a multi-cycle photoelectron spectra (see Supplementary Section III for a full description). The ionizing laser pulse polarization is horizontal in all figures. For parallel and perpendicular alignment the holographic fringes are perfectly symmetric around the horizontal axis. Hence, the ND spectrum is symmetric and, in fact, shows no fringes. At an alignment of $45^{\circ}$ with respect to the ionizing field, the holographic interference is asymmetric, as evidenced in Fig. 3b. Although we do not expect the 2D model to reproduce the quantitative details of the experiment on real $3 \mathrm{D} \mathrm{N} \mathrm{N}_{2}$, the asymmetry is qualitatively captured by our model.

We now seek to explain the origin of the computed and measured fringe shift as a function of alignment angle. To this end, we first investigate field-free scattering of localized wavepackets, constructed to mimic the recollision continuum electron wavepacket. Using the field-free scattering model, we identify properties of the system that lead to the fringe shift. It is found that, in the case of fieldfree scattering at wavepacket parameters comparable to the laserinduced recollision wavepacket, the shift of the fringes reflects a spatial offset between the phase centre of the incident wavepacket and the scattering potential. Following the field-free analysis, we 
revisit the full laser-driven numerical recollision simulations and confirm that the same spatial phase offset is present. From this analysis, we can conclude that the shift of the fringes in the holographic region of the recollision photoelectron spectrum is caused by an effective spatial offset between the phase centre of the continuum wavepacket and the parent ion; the continuum electron wavepacket is not emitted symmetrically about the centre of the molecule, but instead is offset in the direction of the down-field atom when there is an angle between the molecular axis and the electric field.

We use a Gaussian wavepacket for the incident field-free scattering wave

$$
|\mathcal{G}\rangle=\int \mathrm{d} \mathbf{p}|\mathbf{p}\rangle \exp \left[-4 \ln 2\left|\frac{\mathbf{p}-\mathbf{p}_{0}}{\sigma_{p}}\right|^{2}-\mathrm{i} \mathbf{p} \cdot \mathbf{r}_{0}\right]
$$

where $\langle\mathbf{r} \mid \mathbf{p}\rangle=(1 / \sqrt{2 \pi}) \exp [-\mathrm{ip} \cdot \mathbf{r}]$ are the plane-wave momentum states. The wavepacket is characterized by three parameters, the momentum full width at half maximum $\sigma_{p}$, the initial position $\mathbf{r}_{0}=\left(x_{0}, y_{0}\right)$ of the wavepacket at $t=0$, and the average momentum $\mathbf{p}_{0}$. In the laser-driven electron recollision, the continuum wavepacket recollides with energies between zero and 3.17 $U_{\mathrm{p}}=3.17 E_{0}^{2} / 4 \omega^{2}=1.03$ a.u. ( $p_{\max }=1.43$ a.u.). The continuum path length (how far the electron wavepacket travels between ionization and recollision) is of the order of twice the free electron oscillation amplitude in the laser field, one to go away from the core and a second to return to the core, which is given by $2 E_{0} / \omega^{2}=40$ a.u. We choose the Gaussian parameters to be $\left|\mathbf{p}_{0}\right|=1.5$ a.u., $x_{0}=-50$ a.u. and $\sigma_{p}=1$ a.u. to qualitatively mimic the recollision wavepacket. We use the wavepacket in equation (1) as the initial state and numerically propagate across the scattering event using the above numerical model.

Figure 4 shows the results of four different field-free scenarios. The particular geometry in each case is shown in the left column, while the corresponding photoelectron momentum spectrum is shown in the right column. Figure $4 \mathrm{a}$ is for the fully symmetric scattering case where a symmetric wavepacket is incident on a perpendicular molecule. The momentum distribution contains holographic interference fringes analogous to those seen in the field-driven recollision case of Fig. 2a. Note that our field-free wavepacket is narrower in momentum space than for the laserdriven wavepacket, hence the field-free simulations sample a smaller 'spot' of the photoelectron momentum spectra when compared to the laser-driven recollision spectra.

From the general idea of the holographic recollision interference, one might suspect that the fringe shift reflects the angle of the molecule on rescattering. It is then natural to consider scattering of the field-free wavepacket with the molecule at an angle. Figure $4 \mathrm{~b}$ shows the $\theta=45^{\circ}$ case. Although there are very small quantitative differences between the scattered spectra for $\theta=90^{\circ}$ and $45^{\circ}$, varying the angle of the molecule does not result in a significant fringe shift. The reason that the molecular angle $\theta$ has little effect in this scenario is simple-the wavelength of the scattering wavepacket $\lambda=2 \pi /\left|\mathbf{k}_{0}\right|$ is too long to effectively resolve the bond length. This is also the case for the laser parameters used in the recollision simulations above. A peak recollision energy of $3.17 U_{\mathrm{p}}\left(\right.$ at $1.5 \times 10^{14} \mathrm{~W} \mathrm{~cm}^{-2}$ ) means that the smallest recollision wavelength is 4.4 a.u., again too large to effectively resolve the bond length, and hence alignment, of the two-centre potential in the holographic region. In Supplementary Section IV we provide further cases that verify the insensitivity to the bond length and alignment angles. Thus, the holographic fringes, in our parameter range, can not be significantly affected by the rescattering event.

Figures $4 \mathrm{c}, \mathrm{d}$ show scenarios that vary the properties of the incident wavepacket. For these simulations, the two-centre potential
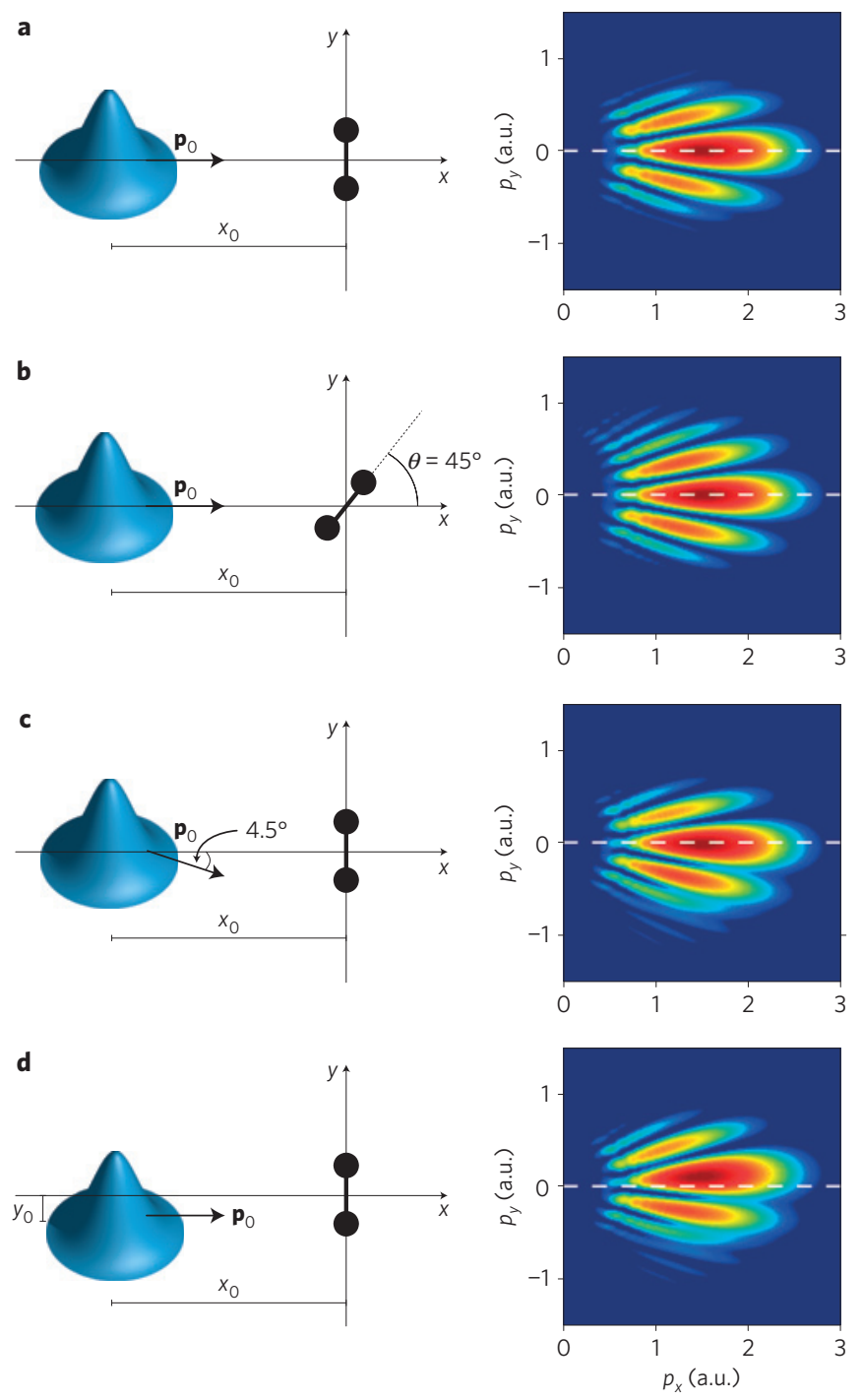

Figure 4 | Four field-free wavepacket scattering scenarios with corresponding final photoelectron spectra showing the holographic fringe pattern for each case. a, Symmetric reference scenario $\left(x_{0}=-50\right.$ a.u., $y_{0}=0$ a.u., $\left|\mathbf{p}_{0}\right|=1.5$ a.u.). b. Two-centre potential set to an angle of $\theta=45^{\circ}$. c, Incident Gaussian wavepacket offset launched at an angle of $-4.5^{\circ}$ below the $+\hat{x}$ axis. $\mathbf{d}$, Incident Gaussian wavepacket offset in the vertical direction ( $y_{0}=-4$ a.u.). The colour scale is logarithmic and covers two orders of magnitude.

was set with the bond axis vertical. However, because the alignment of the bond axis plays little role, any alignment could have been used. In Fig. $4 c, \mathbf{p}_{0}$ was set to point $4.5^{\circ}$ below the $+\hat{x}$ axis, which leads to a vertical drift of the wavepacket centre of -4 a.u. by the time the wavepacket hits the two-centre potential. In this case, no appreciable shift of the fringes develops. In Fig. $4 d$, the initial vertical offset $y_{0}$ was set to -4 a.u., which results in a significant vertical shift of the holographic fringes of same order as the shift seen in the recollision scenario of Fig. 2b. It is useful to recall that the holographic fringes are a result of interference between the scattered wave and the incident wave. Therefore, the most important aspect of the incident wave is a shift along $y$ of the centre curvature of the phase fronts. The fact that centre of the wavepacket amplitude is also shifted along $y$ results in an overall shift of the general envelope that contains the fringes, but effectively does not alter the actual position of the fringes. We can conclude that the shift of the holographic fringes is 

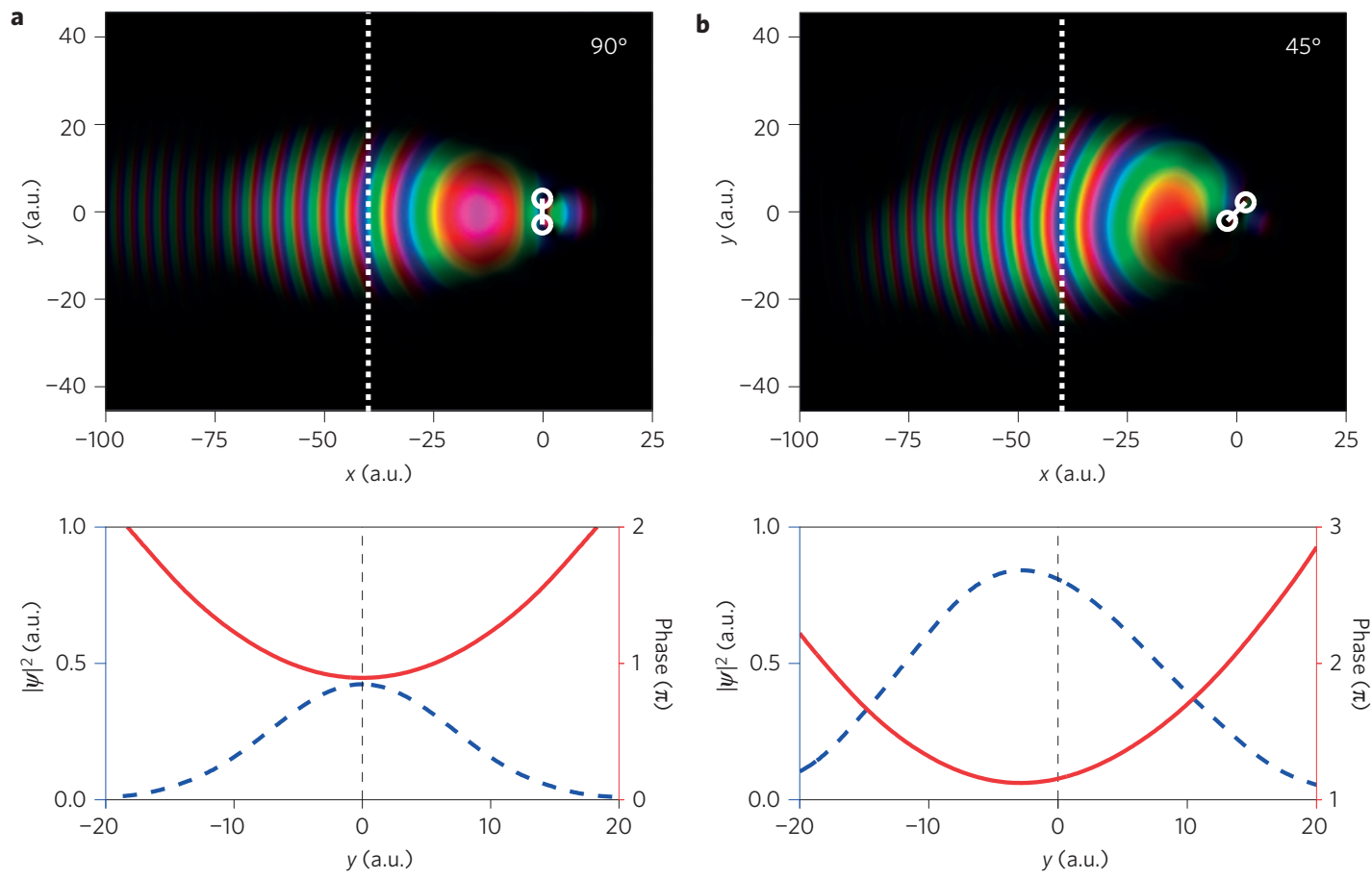

Figure 5 | Simulated two-dimensional continuum electron wavepackets. a, Top panel: wavefunction of the continuum electron in the 2D simulation at time $t=0.625 \tau$ (just before recollision commences) for the $\theta=90^{\circ}$ case. Brightness is the amplitude of the wavefunction (black $=0$ ), while colour shows the complex phase. An (oversized) cartoon depicts the position and angle of the molecule. Bottom panel: line-outs of the amplitude (dashed blue line) and phase (solid red line) at $x=-40$ a.u. (indicated in top panel by a dashed line). b, Same as a but for $\theta=45^{\circ}$.

caused by an effective offset of the centre of phase curvature of the incident wavepacket.

Having identified the cause of the fringe shift in the field-free model, we now analyse the full recollision simulations further to see if a similar mechanism is present. Figure 5a,b shows snapshots of the electronic continuum wavefunctions for the molecular angles $\theta=90^{\circ}$ and $45^{\circ}$, respectively. The snapshots are taken at time $t=0.625 \tau$, where $\tau=2 \pi / \omega$ is the laser oscillation period. At this $t$, the continuum wavefunction is just starting to recollide with the parent core from the left. The top panels show the wavefunction, while the bottom two panels show cuts at $X=40$ a.u. across the recollision wavepacket. For the perpendicular case $\left(\theta=90^{\circ}\right)$, both the phase and the amplitude functions are symmetric around $y=0$. For the $45^{\circ}$ case, neither the phase nor the amplitude is symmetric about $y=0$. Importantly, it is seen that the minimum of the phase in the $45^{\circ}$ case is shifted from $y=0$ by $\sim 3$ a.u., the same order of magnitude of $y$-offset that was needed in Fig. 4 to generate a shifted holographic interference pattern. This phase offset is present for essentially any position $X$, as demonstrated in Supplementary Section VI. We emphasize that, as the holographic fringes are an interference effect, it is the offset of the minimum of the phase from $y=0$ that causes the shift of the holographic pattern. Figure 4 confirms that the shift of the holographic fringes seen in the experimental data reflects asymmetries in the phase of the recollision wavepacket for intermediate molecular angles. These asymmetries of the recollision wavepacket arise in the ionization step as the bound electron escapes across a structured barrier at an angle (for a time-resolved analysis of the ionization step, see Supplementary Section V).

Thus far, we have investigated the source of the holographic fringe shift using the $2 \mathrm{D}$ soft-core model with a bond length of $R_{0}=4$ a.u. As noted above, this is not the correct bond length for $\mathrm{N}_{2}$. If the correct $\mathrm{N}_{2}$ bond length $\left(R_{0}=2 \mathrm{a}\right.$.u. $)$ is used in the 2D model, no substantial angle dependence of the holographic fringes is seen. However, in addition to a lack of shifted fringes, the $2 \mathrm{D}$ model with $R_{0}=2$ a.u. also shows no appreciable offset of the centre of phase curvature of the continuum wavepacket (Supplementary Section VI). Therefore, this leaves us with the question of whether the conclusions drawn from the analysis of the soft-core model are at all relevant to real $3 \mathrm{D} \mathrm{N}_{2}$. To address this question, we now consider a full $3 \mathrm{D}$ model for strong-field ionization of realistic $\mathrm{N}_{2}$ using the time-dependent resolution-in-ionic-states (TD-RIS) method of ref. 24. The TD-RIS method is a numerical approach to calculating molecular strong-field ionization that couples multi-electron orbital-based electronic structure methods, used to represent the field-free multielectron structure, to Cartesian grids to represent the continuum electron. This treatment has the correct spatial dimensionality, has the correct equilibrium bond length of the neutral molecule, uses the correct shape of the initial orbital, and contains the correct ionic electrostatic potential through which the liberated electron escapes. Although the method is capable of including simultaneously ionization to multiple cation states, in the present computation we consider only ionization to the ground state of the cation, which is the dominant channel for strong-field ionization of $\mathrm{N}_{2}$ at the present experimental intensities. A converged computation of the full $3 \mathrm{D}$ photoelectron spectrum is not attainable with the present version of the implemented codes for TD-RIS. However, we can compute the continuum wavepacket after the initial ionization event, analogous to the $2 \mathrm{D}$ results presented in Fig. 5. Figure 6 shows the computed continuum wavefunctions for the perpendicular and $45^{\circ}$ scenarios for the $3 \mathrm{D}$ model. As can be seen, there is a shift of the centre of phase curvature of about $y=-1.45$ a.u. when the molecule is at an angle of $45^{\circ}$. This shift of the curvature is present across the full continuum wavepacket (Supplementary Section VII). Therefore, the origin of the shifted holographic fringes in our $2 \mathrm{D}$ model is also present in the full $3 \mathrm{D}$ modelling of $\mathrm{N}_{2}$. 

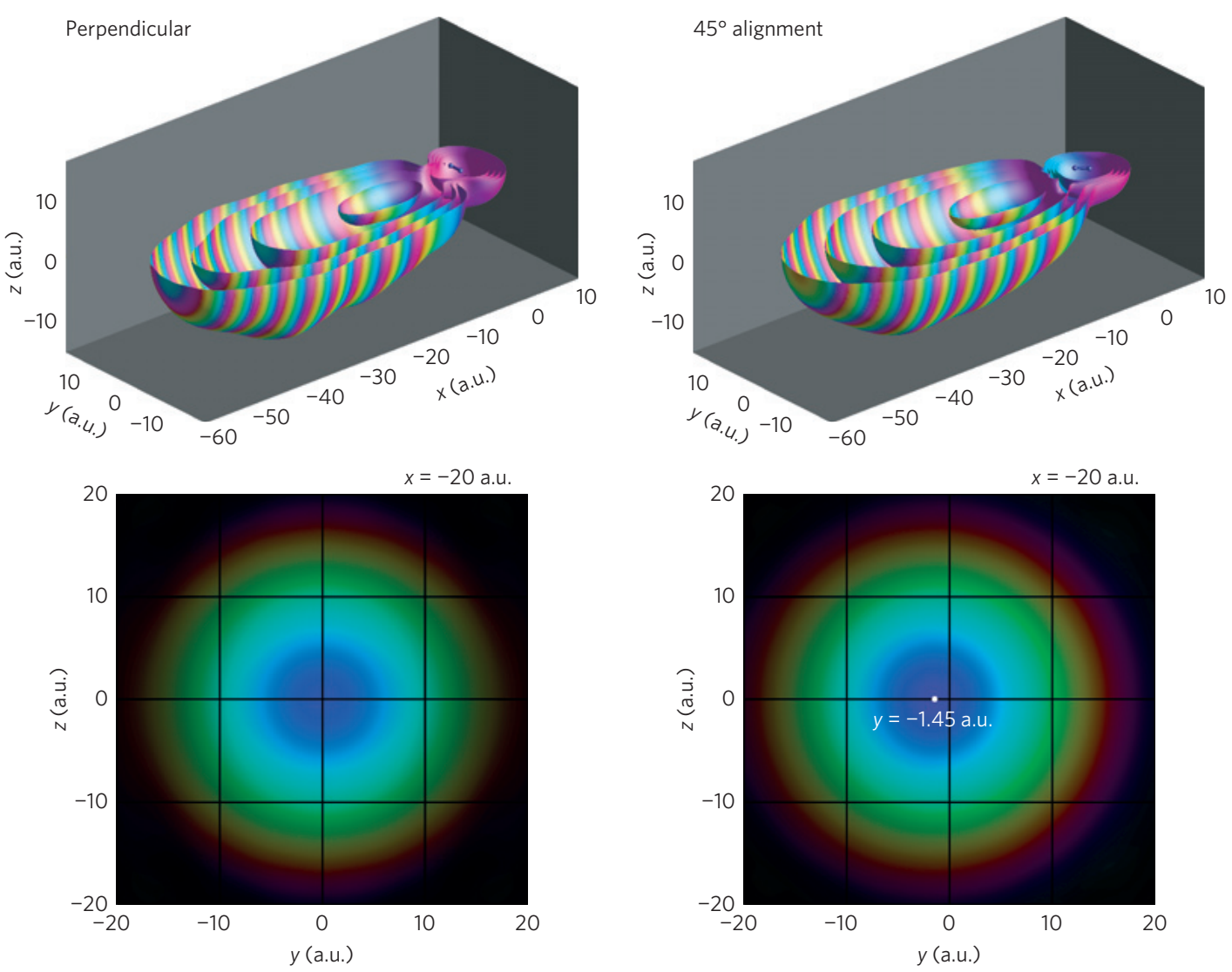

Figure 6 | Simulated three-dimensional continuum electron wavepackets. Wavefunctions and phase cuts for the 3D $\mathrm{N}_{2}$ simulations at time $t=0.5 \tau$, that is, half the laser period. The phase cuts were taken at $x=-20$ a.u. In the $45^{\circ}$ case, the centre of the phase curvature is located at $y=-1.45$ a.u.

Our finding suggests that accurate theoretical descriptions of strong-field recollision physics in molecules will require a structured continuum wavepacket that depends on the orientation of the core-strong-field recollision in molecules is not a simple molecule-centred partial-wave diffraction experiment. Specifically, this impacts the analysis of alignment-dependent high-harmonic radiation, for example, in the tomographic reconstruction of orbitals. In addition, the observed phase offset will contribute to the ionization phase that appears in semi-classical models of highharmonic generation ${ }^{25}$. There is nothing particularly special about the $\mathrm{N}_{2}$ molecule and we expect that analogous phase structures of the continuum wavepacket will be found in strong-field ionization of any molecular system where the laser polarization does not coincide with axes of symmetry of the molecule. In general, the electron wavepacket released from any non-symmetric system via strong-field ionization will exhibit an offset in the phase front that is on the scale of, or larger than, the system itself. Looking ahead, careful measurements of the holographic region of the photoelectron spectra together with accurate theoretical tools could lead to the complete characterization-both amplitude and phaseof the recollision electron in strong-field physics.

Received 30 August 2013; accepted 29 May 2014; published online 20 July 2014

\section{References}

1. Cowley, J. M. Twenty forms of electron holography. Ultramicroscopy 41, 335-348 (1992).

2. Gabor, D. A new microscopic principle. Nature 161, 777-778 (1948).

3. Corkum, P. B. Plasma perspective on strong field multiphoton ionization. Phys. Rev. Lett. 71, 1994-1997 (1993).
4. Huismans, Y. et al. Time-resolved holography with photoelectrons. Science 331, 61-64 (2011).

5. Bian, X-B. et al. Subcycle interference dynamics of time-resolved photoelectron holography with midinfrared laser pulses. Phys. Rev. A 84, 043420 (2011).

6. Marchenko, T., Huismans, Y., Schafer, K. J. \& Vrakking, M. J. J. Criteria for the observation of strong-field photoelectron holography. Phys. Rev. A 84, 053427 (2011).

7. Hickstein, D. D. et al. Direct visualization of laser-driven electron multiple scattering and tunneling distance in strong-field ionization. Phys. Rev. Lett. 109, 073004 (2012).

8. Huismans, Y. et al. Scaling laws for photoelectron holography in the midinfrared wavelength regime. Phys. Rev. Lett. 109, 013002 (2012).

9. Bian, X-B. \& Bandrauk, A. D. Attosecond time-resolved imaging of molecular structure by photoelectron holography. Phys. Rev. Lett. 108, 263003 (2012).

10. Walker, B. et al. Precision measurement of strong field double ionization of helium. Phys. Rev. Lett. 73, 1227-1230 (1994).

11. L'Huillier, A. \& Balcou, Ph. High-order harmonic generation in rare gases with a 1-ps 1053-nm laser. Phys. Rev. Lett. 70, 774-777 (1993).

12. Krausz, F. \& Ivanov, M. Yu. Attosecond physics. Rev. Mod. Phys. 81, 163-234 (2009).

13. Lewenstein, M., Balcou, Ph., Ivanov, M. Yu., L'Huillier, A. \& Corkum, P. B. Theory of high-harmonic generation by low-frequency laser fields. Phys. Rev. A 49, 2117-2132 (1994).

14. Bashkansky, M., Bucksbaum, P. H. \& Schumacher, D. W. Asymmetries in above-treshold ionization. Phys. Rev. Lett. 60, 2458-2461 (1988).

15. Brabec, T., Ivanov, M. Yu. \& Corkum, P. B. Coulomb focusing in intense field atomic processes. Phys. Rev. A 54, R2551 (1996).

16. Muth-Böhm, J., Becker, A. \& Faisal, F. H. M. Suppressed molecular ionization for a class of diatomics in intense femtosecond laser fields. Phys. Rev. Lett. 85, 2280-2283 (2000).

17. Meckel, M. et al. Laser-induced electron tunneling and diffraction. Science 320, 1478-1482 (2008).

18. Akagi, H. et al. Laser tunnel ionization from multiple orbitals in HCl. Science 325, 1364-1367 (2009). 
19. Holmegaard, L. et al. Photoelectron angular distributions from strong-field ionization of oriented molecules. Nature Phys. 6, 428-432 (2010).

20. Ivanov, M. Yu., Brabec, T. \& Corkum, P. B. Coulomb corrections and polarization effects in high-intensity high-harmonic emission. Phys. Rev. A 54, 742-745 (1996)

21. Comtois, D. et al. Observation of coulomb focusing in tunnelling ionization of noble gases. J. Phys. B 38, 1923 (2005).

22. Le, A-T., Lucchese, R. R., Tonzani, S., Morishita, T. \& Lin, C. D. Quantitative rescattering theory for high-order harmonic generation from molecules. Phys. Rev. A 80, 013401 (2009).

23. Ullrich, J. et al. Recoil-ion and electron momentum spectroscopy: Reaction-microscopes. Rep. Prog. Phys. 66, 1463 (2003).

24. Spanner, M. \& Patchkovskii, S. One-electron ionization of multielectron systems in strong nonresonant laser fields. Phys. Rev. A $\mathbf{8 0}$ 063411 (2009).

25. Mairesse, Y. et al. High harmonic spectroscopy of multichannel dynamics in strong-field ionization. Phys. Rev. Lett. 104, 213601 (2010)

\section{Acknowledgements}

R.D. acknowledges support from DAAD and DFG.

\section{Author contributions}

M.M., A.S., D.M.V., P.B.C. and R.D. conceived and designed the experiments. M.M. and A.S. performed the experiments. M.M., A.S. and R.D. analysed the experimental data. M.S. and S.P. built the theoretical codes and analysis tools. M.S. provided the

interpretation of the experimental data and performed the theoretical modelling. All authors contributed to the writing of the manuscript.

\section{Additional information}

Supplementary information is available in the online version of the paper. Reprints and permissions information is available online at www.nature.com/reprints.

Correspondence and requests for materials should be addressed to M.S.

\section{Competing financial interests}

The authors declare no competing financial interests. 